\title{
MedlinePlus at 21: A website devoted to consumer health information
}

\author{
Terry Ahmed* \\ Heat, Reference \& Web Services, National Library of Medicine, USA
}

\begin{abstract}
This report is on the consumer health website, MedlinePlus. This website was created by the National Library of Medicine and features content produced by the National Institutes of Health. This report covers an overview, origin, content and possible future evolution of this website. The report also spotlights the specific features of the MedlinePlus health topic on health literacy and covers in more detail the PubMed Topic Specific Query on health literacy.
\end{abstract}

Keywords: Health literacy, MedlinePlus, consumer health, National Library of Medicine, National Institutes of Health

\section{Introduction}

This report is organized into six sections which provide a brief overview of MedlinePlus.gov; discuss its creation; note its audience and inclusion of content guidelines; explain the website's health literacy health topic page; introduce the free MEDLINE/PubMed search of health literacy literature; mention MedlinePlus' recent and future changes; and provide a conclusion. Some past and present efforts to advance health literacy within MedlinePlus.gov are mentioned.

\section{MedlinePlus: Brief overview}

The U.S. National Library of Medicine (NLM) launched MedlinePlus.gov in October 1998. Since its first day about 21 years ago, MedlinePlus has provided health information intended for health consumers, or general audiences. MedlinePlus.gov attempts to enhance the public's understanding of health and medicine, so the website might be considered as a working experiment to promote health literacy in English and Spanish and increasingly, in other languages.

MedlinePlus.gov is free of charge and contains no advertising. It is financially supported by the U.S. Congress through its funding of NLM and the National Institutes of Health (NIH). MedlinePlus consists of consumer health information from NIH, other U.S. federal health agencies, and diverse governmental and non-governmental evidence-based health organizations in the U.S. and internationally. MedlinePlus features a comprehensive medical encyclopedia, a drug information database, information

*E-mail: ahmedt@mail.nlm.nih.gov. 
about vitamins and supplements, laboratory test information, and information in multiple languages. MedlinePlus includes health and surgery video materials.

MedlinePlus is available in English and Spanish. A user can toggle into either language on a link on the top right side of the home as well as other pages on the website.

Much of the content on MedlinePlus is organized into 1030 health topic pages in English (and 1027 in Spanish) that cover prevalent diseases and conditions. The health topic pages include health information for vulnerable populations including: African American health; Hispanic American health; Native American health; rural health concerns; Native Hawaiian and Pacific Islander health; Gay, Lesbian, Bisexual, and Transgender health; and Veterans and Military health.

There is an A to Z list of health topic pages to browse through so users can target information and navigate to a specific topic. There also is a search box located on each page that leads to health topic and other pages. Health topic pages additionally are organized generically by: body location/systems; disorders and conditions; diagnosis and therapy; demographic groups; and health \& wellness.

Each health topic page consists of a summary explanation of the topic with relevant information listed below a summary that navigates a user to more in-depth information. The summaries are written for lay audiences and frequently are derived from NIH-developed health information. There are several categories within each health topic. The categories within most health topic pages include: diagnosis and tests; treatment and therapies; related issues; genetics; statistics and research; clinical trials; and others. These categories may vary depending on the topic and length of a health topic page.

Many health topic pages also provide links to patient handouts that come from the Medical Encyclopedia. While physicians are encouraged to print handouts for patients, anyone can print handouts for personal, family, or caregiver use. The Medical Encyclopedia includes more than 4,000 articles about diseases, conditions, tests, symptoms, injuries, and surgeries. In addition, it contains an extensive library of medical photographs and illustrations. The encyclopedia articles are listed in alphabetical order within an $\mathrm{A}$ to $\mathrm{Z}$ list. Specific articles can also be searched for using the search box. The Medical Encyclopedia is not curated by NLM; it is purchased content published by A.D.A.M. Inc.

The Drugs, Herbs and Supplements section of MedlinePlus section is divided into drugs and herbs and supplements. The drug section includes information on prescription and over-the-counter drugs, which is provided by the American Society of Health System-Pharmacists (ASHP). The Herbs and Supplement information is provided by the U.S. National Center for Complementary and Integrative Health and the U.S. Natural Medicines Comprehensive Database. The latter includes dietary supplements and herbal remedies, so users can learn about their effectiveness, usual dosage, and drug interactions.

MedlinePlus also includes laboratory test information where users can learn more about personal laboratory tests, including what a lab test is used for, why a doctor ordered it, how the test will feel, and how to assess the results.

A videos \& tools section provides programs on topics such as anatomy, body systems, and surgical procedures. Users can test their knowledge with interactive tutorials and games. From a staff perspective, one of the surprises regarding MedlinePlus.gov's use has been the enduring popularity of videos of surgeries.

MedlinePlus.gov had about 84 million unique visitors and 179 million page visits from October through December 18, 2018 [1]. By comparison - during its initial quarter (October-December 1998), MedlinePlus received about 53,000 unique visitors and about 682,000 page visits [1]. User statistics that span MedlinePlus.gov's history are available at: https://medlineplus.gov/usestatistics.html. An outline of MedlinePlus.gov's milestones are available at: https://medlineplus.gov/milestones.html [2]. 


\section{MedlinePlus' creation}

Prior to MedlinePlus.gov's launch, NLM's services primarily were devoted for use by physicians, researchers, and librarians. Health consumers increasingly discovered NLM as its web presence for health care professionals evolved through services such as PubMed, which was launched in 1996. PubMed provides access to research published in international, refereed medical journals [3].

As consumer access to the Internet became more common in the 1990s, NLM's audience for thenprofessionally oriented services began to attract members of the general public, often including patients or representatives of the families of patients.

Initially, the public's interest was observable via increasing consumer inquiries that NLM's customer service department received. Inquiries began to increase from across the U.S. and internationally in the early to mid-1990s. The questions from non-health professionals included inquiries about diseases, wellness, treatment, locating a physician, locating a hospital and drug information, and sometimes finding health information. NLM's reference librarians often directed persons to then-available Internet (or storedlibrary) resources to either find or research an answer to individual queries. As the latter increased, the need (and opportunity) for a consumer-oriented health information service became increasingly apparent to NLM's medical librarians and senior staff.

In one of its pre-MedlinePlus launch efforts, NLM completed a pilot project involving 39 public library organizations with more than 200 locations in nine U.S. states (Alabama, Georgia, Maryland, New York, Pennsylvania, South Carolina, Tennessee, Texas and Virginia and the District of Columbia). The Medical Questions project was designed to increase public awareness of - and access to - health information via the Internet [4]. Albeit intrinsically valuable, the Medical Questions initiative helped motivate NLM to find a new way to create a resource that could interactively assist with ongoing consumer inquiries about health and medicine - and provide an always available, alternative resource to answer public questions (that did not rely on the limited availability of NLM's medical librarians).

During the time MedlinePlus was planned, developed, and launched, NLM's then-director (Donald A.B. Lindberg M.D.) often reminded the project's staff that: (a) informed consumers are better patients and (b) patients, caregivers, and non-medical professionals have diverse health information needs. Fortunately, the MedlinePlus.gov development team agreed the then-nascent health information service should address these as well as other consumer health information challenges, which included providing access to understandable (or health literate) medical information.

From the outset, MedlinePlus was developed as an easy-to-understand resource for the public. While the term 'health literacy' was not widely used in the mid-1990s, the need to improve the public's understanding of health (that included helping patients comprehend complex medical jargon and terminology) was internally acknowledged as part of MedlinePlus' mission.

A second challenge was to provide a new route for the public to access MEDLINE and NIH's comprehensive and authoritative health information resources. Hence, a comprehensive consumer health portal was created with access to MEDLINE (the world's largest database of peer-reviewed medical literature). Eventually, this was coupled with links to NIH's other consumer health information resources, clearinghouses from non-governmental and governmental health-related organizations, clinical trials, and even consumer self-help medical groups.

A third challenge was to meet a pressing, ongoing need for health information to be current, updated, reviewed regularly, and clinically valid (or as evidence-based as possible).

To respond to all these challenges, NLM combined the enduring skills of medical librarians and then-pioneering health information technology experts to jointly curate the new, comprehensive, health 
information service. For more than two decades, MedlinePlus' depth and diversity have depended on the skills of medical librarians and health information technology experts to organize, collect, classify, and provide health information to the public. MedlinePlus.gov's utility remains dependent on the latter coordination with an understanding that MedlinePlus should strive to respond to (and anticipate) consumer health needs by providing comprehensive and understandable medical information.

\section{MedlinePlus' audience and inclusion of content guidelines}

The primary audience for MedlinePlus is the 'public,' which is operationalized as patients and families of patients. These populations were identified as having the greatest need for evidence-based medical information in the aforementioned Medical Questions initiative [4]. Indeed, for the past 21 years, the needs of patients and families of patients have been considered first and foremost in producing, curating, or changing MedlinePlus' content. A prevailing interest is to help patients and families of patients find authoritative health information from a comprehensive web portal.

MedlinePlus.gov keeps track of the ongoing international and domestic use of the service. Using analytics tools, MedlinePlus.gov's staff can determine usage by geolocation. For example, North America is the heaviest usage area, followed by Asia, Oceania, and Africa. Within the U.S., more MedlinePlus users are clustered in these high population U.S. states: California; Texas; New York; Florida; Illinois; Pennsylvania; Georgia; Ohio; North Carolina; and Michigan. Visitors to NLM can view monitors that update the geographical dispersion of interest in MedlinePlus as well as some of NLM's other health information technology services.

Today, MedlinePlus.gov provides free access to information produced by NLM and NIH, such as searches of MEDLINE/PubMed, and ClinicalTrials.gov (the database of clinical research studies conducted at the National Institutes of Health and clinical institutions worldwide).

MedlinePlus adheres to strict quality guidelines that determine what health and clinical information is provided. While most evidence-based information comes from NIH institutes, additional sources include other federal agencies and some nongovernmental health organizations.

MedlinePlus.gov's quality guidelines, or whether MedlinePlus links to a candidate website's health information, are based on the following criteria: the quality, authority and accuracy of the provided health content; a candidate website's advertising guidelines; and the availability and maintenance of a candidate's website.

Some of the specific criteria within each of these areas are provided below.

\subsection{Quality, authority and accuracy of health content}

- In order to be linked, the mission of the organization that provides health information must reflect MedlinePlus' educational goals and intended audience.

- To be linked, a candidate organization must provide accurate, evidence-based information that complements or enhances the government information found in MedlinePlus.

- The source of the candidate's website's content must be established, respected, and evidence-based. A candidate organization needs to publish a list of advisory board members or consultants to their website.

- The information provided on the candidate's website needs to be appropriate to MedlinePlus' audience's reading level, well-organized, and easy to use. (The latter criterion operationalizes some health literacy principles) [5]. 
- Candidate health websites should provide original content.

- Candidate health websites should have a process to review the availability and evidentiary foundation of all provided information and links.

4.2. The primary purpose of a candidate website should be educational and not to sell a product or service. Most content should be available at no charge

- MedlinePlus prefers no advertising on candidate web pages. In the infrequent case where advertising is displayed, the advertising should not suggest a commercial influence on the selection and production of health content.

- The candidate website needs to clearly differentiate between its health content and advertising. There should be an advertising policy on the site. Advertisers or sponsors must not play a role in selecting or editing health information.

- MedlinePlus excludes organizations and web resources if the presentation or content might lead a reasonable user to infer an endorsement of health products or services.

- While MedlinePlus provides links to directories to help find health professionals, services, and facilities, NLM does not endorse or recommend the organizations that produce directories, or the individuals and organizations included in the directories.

\subsection{Availability and maintenance of a candidate website}

- The candidate website should be available consistently and lack technical usability issues.

- The candidate website should link only to reliable sources and maintain the links.

- The source for the contents on the candidate's web page(s) and the entity responsible for maintaining the website (e.g. the webmaster, organization, creator of the content) need to be transparent.

- The information on a candidate website should be current, or an update timeline should be included.

- User registration should not be required to view the information on a candidate's website.

Turning to MedlinePlus' interest in advancing consumer readability, the information on MedlinePlus, such as the summaries on the health topic pages, are written at the fifth to eight grade reading level [6]. MedlinePlus also curates and identifies an easy-to-read collection of health information provided by diverse health institutes and agencies: https://medlineplus.gov/all_easytoread.html.

To encourage other health communicators, NLM provides instructions/guidance about how to write easy-to read health materials: https://medlineplus.gov/etr.html. The link lists step-by-step instructions to create easy-to-read contributions.

To help physicians and health care professionals provide patient materials, MedlinePlus' Patient Handout information is provided in the website's medical encyclopedia. Health professionals can easily print this information and provide it to their patients. MedlinePlus contains thousands of pages identified as patient handouts, which often are available within health topic pages.

Since 2002, MedlinePlus has been available in Spanish. The health topic pages in Spanish are translated directly from the English version. When links are bilingual, they are provided in both languages. Some links to health information in Spanish are available only in the Spanish language. Otherwise, the health information links on Spanish topic pages either utilize trusted NIH resources, or health organizations whose content follows the aforementioned inclusion criteria. 
In addition to 1027 Spanish language health topic pages and other information en espanol, MedlinePlus includes other multilingual resources of health information. The process to select health services in other languages is explained in: https://medlineplus.gov/languages/criteria.html [7].

The multilingual resources on MedlinePlus often are derived from the HealthReach collection at https://healthreach.nlm.nih.gov/ [8]. HealthReach identifies, collects, and makes available free resources that are accurate, up-to-date, and assessed for quality. A caveat; MedlinePlus' multilingual collection is not as comprehensive as its health resources in English and Spanish. In some grey areas, such as health resources specific to refugee resettlement and education, some services may be integrated in HealthReach but not within MedlinePlus.gov.

\section{Health literacy health topic, MEDLINE/PubMed search, and health literacy information resources}

MedlinePlus has a health topic page devoted to help consumers understand health literacy: https://medlineplus.gov/healthliteracy.html [9]. The topic page consists of a summary about health literacy and lists (and links to) many related resources.

While the information in MedlinePlus' health literacy health topic page is geared towards general users, it provides an overview of the field for health professionals. Currently, the listed health literacy resources include information from: NIH; the U.S. Department of Health and Human Services (HHS); the U.S. Centers for Disease Control and Prevention (CDC); and the U.S. Food and Drug Administration (FDA). Similar to others, the health topic page is organized into different subcategories so users easily can locate specific health literacy information. The topic page also lists related resources from other areas within MedlinePlus, which incorporates information from the medical encyclopedia, related health topics, and information in other languages.

As a compliment to the consumer-oriented health literacy topic page in MedlinePlus, NLM maintains the MEDLINE/PubMed Search and Health Literacy Information Resources resource page: https://www.nlm.nih.gov/services/queries/health_literacy.html [10]. The latter resource includes a comprehensive literature search of PubMed that retrieves references about health literacy and provides access to health literacy research that has been published in international medical journals. Users can click on the search link to launch the search and review the results.

The health literacy search strategy is reviewed periodically by NLM medical librarians; it evolved from an early 21st century effort to assist the then-new National Academy of Sciences, Engineering, and Medicine Roundtable on Health Literacy [11]. The health literacy search engine incorporates many of the subfields within health literacy scholarship.

The current search parameters are:

(health[ti] AND literacy[ti])

OR ("health literacy" OR "health literate" OR "medical literacy")

OR (functional[tw] AND health[tw] AND literacy[tw])

OR numeracy

OR ((low literate[ti] OR low literacy[ti] OR literacy[ti] OR illiteracy[ti] OR literate[ti] OR illiterate[ti]

OR reading[mh] OR comprehension[mh]) AND (health promotion[major] OR health education[major]

OR patient education[major] OR Communication Barriers[major] OR communication[major:noexp]

OR Health Knowledge, Attitudes, Practice[major] OR attitude to health[major])) OR

(comprehension[major] AND educational status[major]) 
OR (family[ti] AND literacy[ti])

OR ((“drug labeling" OR Prescriptions [mh]) AND (“comprehension" OR "numeracy"))

OR ((cancer[ti] OR diabetes[ti]) AND (literacy[ti] OR comprehension[ti]))

OR "adult literacy"

OR "limited literacy"

OR "patient understanding" [ti]

OR (self care [major] AND perception[mh])

OR (comprehension AND food labeling[mh])

OR (comprehension AND informed consent)

OR (comprehension AND insurance, health)

AND English[la]

The health literacy search strategy is comprehensive. Users who are familiar with PubMed can alter the above terms by substituting or adding terms to limit the results to suit individual needs. Instructions on how to use PubMed are available at: https://www.nlm.nih.gov/bsd/disted/pubmedtutorial/cover.html.

The aforementioned, broader resource page also lists links to a range of health literacy information resources from NLM and NIH and includes links to websites provided by international governmental and nongovernmental agencies as selected by NLM's medical librarians.

\section{MedlinePlus' evolution and future}

MedlinePlus.gov began on the Internet as a curated list of links to consumer health information produced primarily by NIH websites. While the website's scope has expanded significantly since 1998, MedlinePlus.gov also made at least four technical changes in the 21 st century that advanced the website's interface and utility. These changes included: a migration from a portal to a search engine model; the use of responsive design; the introduction of MedlinePlus Connect (that links MedlinePlus' information with electronic health record platforms); and the introduction of an extensible markup language (XML) format. Each will be briefly introduced with a discussion of MedlinePlus.gov's future as a repository of health data.

When MedlinePlus began, many Internet developers used a portal model to provide information to users, where information was distributed among 'portal' sites. Portal sites often organize content by subject and user search for a category of interest and then, proceed to narrow a search based on subject identifiers. MedlinePlus adopted a portal architecture at the website's launch, which has migrated in recent years to a search engine model.

Although portal models continue to be a viable source of health information for consumers, an improved search capacity and interface has provided an alternative interface architecture during the past 10-15 years. The evolution of search engines, such as Google, Bing and others, created an interface where a user could type in a plain language term and receive increasingly tailored links to highly relevant information. Some search engines even display a text summary of the answer to specific inquiries. While search engines have been available on websites since the Internet's inception, an improved responsive capacity to search literature and respond more precisely to plain language inquiries have generated a transition to a search engine model.

Currently, MedlinePlus seeks to reach users by structuring the website's content to be more locatable and work in better tandem with advanced search engines, which requires the adoption of a search engine 
model. Perhaps a migration to a search engine model is fitting since MedlinePlus originally was developed as an alternative, aforementioned option for consumers to obtain answers to health questions.

Second, in November 2010, NLM introduced MedlinePlus Connect, a free service that enables any electronic health record (EHR) system to link to relevant, authoritative and current health information from MedlinePlus. MedlinePlus Connect delivers information about conditions and disorders, health and wellness, as well as prescription and over-the-counter medications to patients, families, and health care providers as needed by incorporating MedlinePlus' content into diverse EHR systems.

MedlinePlus Connect works by accepting specific requests from EHR systems and then, provides links to the relevant health information in MedlinePlus. To accomplish this, NLM mapped all MedlinePlus health topic pages to the standard diagnostic coding systems used in EHRs. Specifically, NLM used the ICD-9-CM (International Classification of Diseases, 9th edition, Clinical Modification) and the SNOMED CT CORE Problem List Subset (Systematized Nomenclature of Medicine, Clinical Terms, CORE Problem List Subset) as the code mapping foundations.

MedlinePlus Connect also links to medication information using patient friendly language. When EHR systems send MedlinePlus Connect a request containing a standardized medication code, the service returns links to the most appropriate drug information for prescription and over-the-counter medicines. For medication requests, MedlinePlus Connect supports RXCUI (RxNorm Concept Unique Identifier) and NDC (National Drug Code), which are widely used in the U.S.

Third, in 2015 MedlinePlus switched from a separate desktop and mobile version of the website to a responsive web design with an identical and simultaneous display across diverse Internet platforms. Responsive web design optimizes user interaction by adjusting each page based on the device used regardless if it is a desktop monitor or a small mobile touchscreen. Incidentally, this change was preceded by increasing information that most users linked to MedlinePlus via mobile devices rather than desktop computers.

Fourth, in September 2010 NLM released a search-based web service that provides access to MedlinePlus' health topic data in extensible markup language (XML) format. This change enables software developers to build applications that incorporate health information provided by MedlinePlus. Via this process, MedlinePlus data can be transformed into a more customizable view for specific user populations.

MedlinePlus continues to evolve so it can better present selected consumer health information from NIH and other governmental and non-governmental sources. In the future, MedlinePlus.gov could become more of a repository of health data provided by medical journals and organizations. This prediction is based on the International Committee of Medical Journal Editors' (ICMJE) recent decision to ask future authors to plan to deposit the data sets that underlie the findings within a published study [12]. ICMJE's request means someday the electronic version of medical journals may include vast health data sets that will be accessible to readers. As a result, MedlinePlus users would be able to view the details that undergird some medical research in heretofore unavailable detail.

While future data set availability is sometimes perceived as transformative by medical researchers, the impact on consumers remains more difficult to predict because of the complexity and curated accuracy of the health data [12]. So, a new challenge for NLM and MedlinePlus will be to help consumers make sense of vast, new repositories of medical research information and perhaps someday use these services as a resource to answer individual medical questions. 


\section{Conclusion: How MedlinePlus advances health literacy}

MedlinePlus.gov helps advance health literacy by adhering to a strict policy to provide links to consumer health information written for lay users. MedlinePlus.gov is not targeted toward researchers or health professionals and avoids using medical jargon or terms commonly exercised in the medical research community. Written at a fifth to eight grade reading level, MedlinePlus is for patients and friends/families of patients. MedlinePlus encourages communicators to use plain language to explain health and medicine. The website also links to succinct, clear, and patient-friendly materials available on the Internet.

Besides a topic page devoted to health literacy, MedlinePlus adds health topic pages that seek to improve numeracy and the public's understanding of medical research. These include: understanding medical research https://medlineplus.gov/understandingmedicalresearch.html and evaluating health information https://medlineplus.gov/evaluatinghealthinformation.html $[13,14]$. Each of the latter health topic pages provide an array of links about health research and numeracy related resources, which are written for general audiences.

MedlinePlus also helps patients and caregivers with a limited understanding of medical jargon locate relevant information. Similarly, MedlinePlus seeks to bridge comprehension gaps by: furnishing definitions of medical terms; helping explain the data in routine laboratory tests; emphasizing easy-tounderstand medical information; and providing questions for patients to ask physicians during office visits for some diseases and conditions.

Overall, MedlinePlus strives to help patients and caregivers navigate the health care delivery system, understand individual, family, and public health, and build a body of knowledge to better take care of oneself, friends, family, and even communities. Ultimately, MedlinePlus.gov's goal is to ease consumer access to evidence-based health information, which helps improve the quality of life of adults, teens, toddlers, and citizens. As such, MedlinePlus.gov remains a working experiment to improve the public's understanding of health and medicine and enhance health literacy in the U.S. and around the world.

\section{Acknowledgements}

Finally, the author pays tribute to the NLM staff members and contractors who have worked on MedlinePlus and embraced its consumer health-oriented mission in the 21 years since the website's inception.

\section{References}

[1] MedlinePlus [Internet]. Bethesda, MD: National Library of Medicine; MedlinePlus Statistics; [cited 2019 Feb 27] Available from: https://medlineplus.gov/usestatistics.html.

[2] MedlinePlus [Internet]. Bethesda, MD: National Library of Medicine; MedlinePlus Milestones: 1998-present; [cited 2019 Feb 25] Available from: https://medlineplus.gov/milestones.html.

[3] National Library of Medicine. PubMed [Internet]. Bethesda, MD: 2019 [cited 2019 Feb 25] Available from: https://www.ncbi.nlm.nih.gov/pubmed.

[4] F.B. Wood, B. Lyon, M.B. Schell, P. Kitendaugh, V.H. Cid and E.R. Siegel, Public library consumer health information pilot project: Results of a National Library of Medicine evaluation, Bull Med Lib Assoc 88(4) (2000), 314-322.

[5] R.M. Parker and S.C. Ratzen, Health literacy: A second decade of distinctions for Americans, J Health Commun 15(Suppl 2) (2010), 20-33, doi:10.1080/10810730.2010.501094. R.A. Logan, Health literacy research. in: Meeting Health Information Needs Outside of Healthcare: Opportunities and Challenges, C. Arnott-Smith and A. Keselman (eds), Chandos, Waltham, MA, 2015, pp. 19-38. 
[6] MedlinePlus [Internet]. Bethesda, MD: National Library of Medicine; Easy To Read [cited 2019 Mar 6] Available from: https://medlineplus.gov/faq/easytoread.html.

[7] MedlinePlus [Internet]. Bethesda, MD: National Library of Medicine; MedlinePlus Quality Guidelines for Health Information in Multiple Languages [cited $2019 \mathrm{Feb}$ 27] Available from: https://medlineplus.gov/languages/criteria.html.

[8] HealthReach [Internet]. Bethesda, MD: National Library of Medicine: 2019 [cited 2019 Feb 27] Available from: https://healthreach.nlm.nih.gov.

[9] MedlinePlus [Internet]. Bethesda, MD: National Library of Medicine; Health Literacy [cited 2019 Feb 27] Available from: https://medlineplus.gov/healthliteracy.html.

[10] National Library of Medicine [Internet]. Bethesda, MD: 2019. MEDLINE/PubMed Search and Health Literacy Information Resources; [cited 2019 Feb 27]. Available from: https://www.nlm.nih.gov/services/queries/health_literacy.html.

[11] The National Academies of Sciences Engineering Medicine [Internet]. Washington, DC; 2019. Roundtable on Health Literacy; [cited 2019 Feb 26]. Available from: http://nationalacademies.org/hmd/Activities/PublicHealth/HealthLiteracy.aspx.

[12] D.B. Taichman, J. Backus, C. Baethge, H. Bauchner, P.W. de Leeuw, J.M. Drazen et al., Sharing clinical trial data: A proposal from the International Committee of Medical Journal Editors, Ann Int Med 164: (2016), 505-506, International Committee of Medical Journal Editors. Recommendations for the conduct, reporting, editing, and publication of scholarly work in medical journals. http://www.icmje.org. Updated December 2017. Retrieved February 26, 2019.

[13] MedlinePlus [Internet]. Bethesda, MD. National Library of Medicine; 2019. Understanding Medicine Research; 2019 Dec 31 [cited 2019 Mar 10] Available from: https://medlineplus.gov/understandingmedicalresearch.html.

[14] MedlinePlus [Internet]. Bethesda, MD. National Library of Medicine; 2-19. Evaluating Health Information; 2019 Feb 7 [cited 2019 Mar 10] Available from: https://medlineplus.gov/evaluatinghealthinformation.html.

[15] City of Milwaukee. Milwaukee succeeds: Early childhood immunization; 2016. https://milwaukeesucceeds.org/what-wedo/kindergarten-readiness/early-childhood-immunization. Retrieved Oct 29, 2018.

[16] J. Gray-Murray, M. Leary, M. Watts, F. Xiong and E. Willis, Field methods for discovering practical wisdom: The microdynamics of going beyond technical rationality in real-world practice, Int Q Community Health Educ 33(1) (20122013), 39-53, doi:10.2190/IQ.33.1.d.

[17] E. Willis, E. Ngui, V. Chen, C. Cronk, J. Meurer, S. Shankle, P. McManus and A. Harvieux, Navigating the complexity of relationships in community-based participatory research, Nova Science Pub 9: (2008), 161-182, NLM ID: 101478480. 